\title{
A Simple Model for the Quantum Hydrodynamic Simulation of Electron Transport in Quantum Confined Structures in the Presence of Vortices
}

\author{
J. R. BARKER* \\ Nanoelectronics Research Centre, Department of Electronics and Electrical Engineering, \\ University of Glasgow, Glasgow G12 8LT, Scotland, UK
}

\begin{abstract}
It is demonstrated that the ballistic quantum transport properties of an open quantum dot may be described by an ensemble of spatially correlated virtual classical particles moving within self-avoiding strings. The string paths correspond to ray trajectories. The strings exhibit the necessary properties of self-avoidance, interference and the non-local condition $\oint m \mathbf{v} \cdot d \mathbf{r}=n h$. The formalism suggests that numerical simulation of quantum flows may be constructed $a b$ initio by using the string representation.
\end{abstract}

Keywords: Quantum transport; Quantum dots; Quantum hydrodynamics; String model

\section{INTRODUCTION}

In a recent study [1] of steady state quantum transport through open quantum dots we have demonstrated that numerical simulations based on the transfer matrix method may be revealingly mapped into velocity flows where the velocity vector field $\mathbf{v}(\mathbf{x})$ is defined as the ratio of the quantum mechanical probability current density $\mathbf{j}(\mathbf{x})$ to the probability density $\rho$. The velocity streamlines do not cross: a property of the singlevaluedness of the wave function which leads to the velocity field being derived from the gradient of the phase of the wave function. Consequently, the qualitative features of the steady flow may be deduced from the singularity structure in the flow which may be shown to comprise vortex centres (generally nodes in the wave function) and hyperbolic points (generally at nodes in the velocity field) (see Fig. 1). This picture is generic but we shall focus on a simple twodimensional Hamiltonian system for simplicity: $\mathrm{H}=\mathbf{p}^{2} / 2 \mathrm{~m}+\mathrm{V}(\mathbf{x})$.

If the quantum state of the system is known, for example, in the polar representation where we know the amplitude $R(x, t)$ and phase $S(x, t)$ of the wave function $\Psi(\mathbf{x}, t)=R(\mathbf{x}, t) \exp [i S(\mathbf{x}, t) / \hbar]$, then we can construct an a posteriori quantum

*Tel.: +44 141330 5221, Fax: +44 141330 4907, e-mail: jbarker@elec.gla.ac.uk 


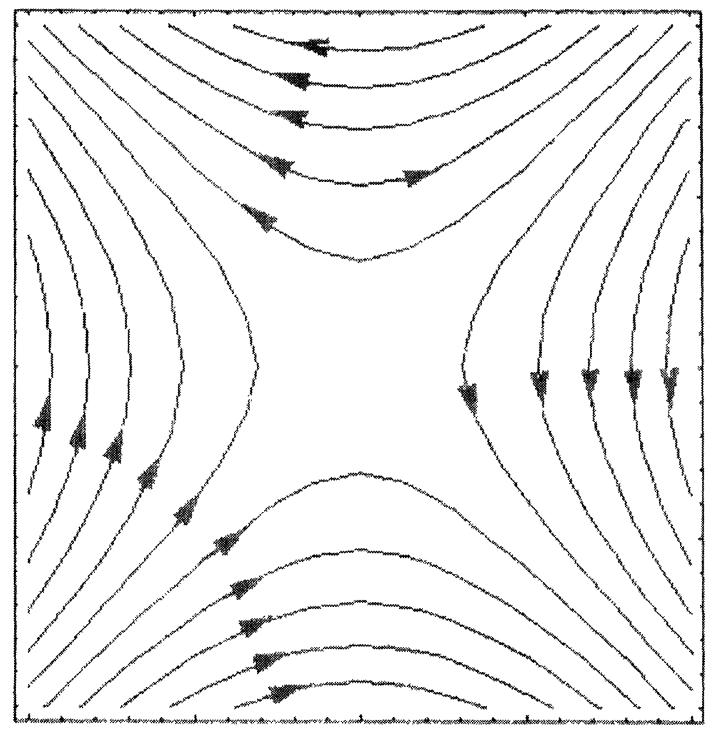

(a)

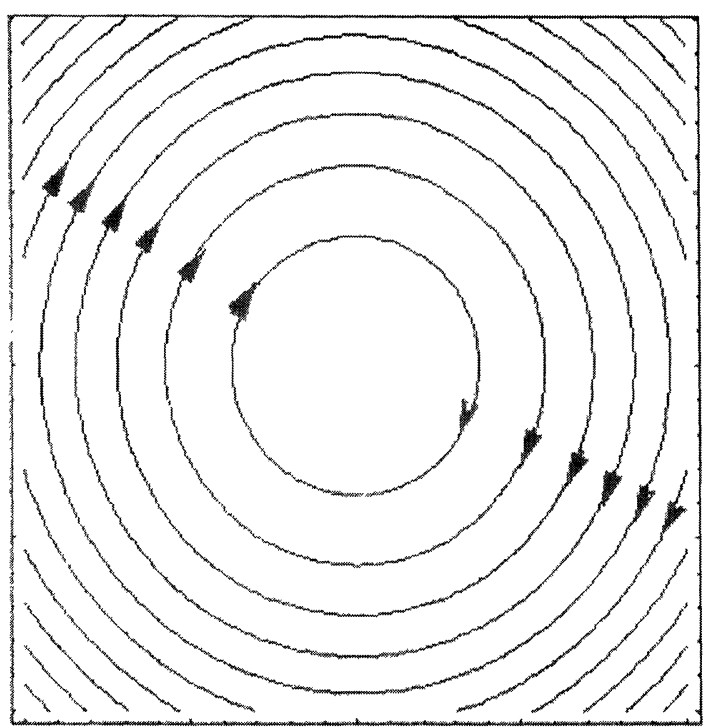

(b)

FIGURE 1 Velocity fields in the vicinity of the singular points of the quantum flows. (a) Hyperbolic fixed point; (b) Vortex centre.

hydrodynamics in which the probability velocity flow and probability density satisfy a continuity equation (Eq. (1)), an Euler like equation which contains the quantum potential (Eqs. $(2,3))$.

$$
\begin{gathered}
\frac{\partial \rho}{\partial t}+\nabla \cdot \rho \mathbf{v}=0 \\
m \frac{\partial \mathbf{v}}{\partial t}+m \mathbf{v} \cdot \nabla \mathbf{v}=-\nabla V(\mathbf{x}, t)-\nabla \mathbf{V}_{\mathbf{Q}}(\mathbf{x}, t) \\
V_{Q}=-\left(\frac{\hbar^{2}}{2 m}\right) \frac{\nabla^{2} R(\mathbf{x}, t)}{R(\mathbf{x}, t)} ; \quad \rho=R^{2} ; \quad m \mathbf{v}=\nabla S(\mathbf{x}, t)
\end{gathered}
$$

A third non-local condition (Eq. (4)) is also satisfied automatically if the wave function is known; it corresponds to the condition that the wave function is single valued and has deep consequences if gauge invariance is taken into account:

$$
\oint \nabla S \cdot d \mathbf{r}=n h(n: \text { integer })
$$

The main purpose of a posteriori quantum hydrodynamics is in its interpretative value.

The construction of an a priori or ab initio quantum hydrodynamics or even a quantum Monte Carlo particle ensemble picture has been an important target in recent years, particularly as Eqs. (1) and (2) seem to provide a simple route to modelling quantum effects in decanano MOSFETs by using the quantum potential (which is a function $\rho$ of only in classical hydrodynamic models). The aim of ab initio quantum hydrodynamics is to use the fluid Eqs. (1) and (2) to construct flows knowing only the appropriate initial conditions and boundary conditions.

However, primarily because of condition (4) which imposes a quantisation of velocity circulation (a matter made more complex for mixed states), it is not possible to reconstruct correctly even simple solutions to the Schrödinger equation $[2,3]$ by $a b$ initio methods. The problem is exacerbated by the difficulty of generating the correct singularities in the flow which indeed are only fixed for stationary flows. The singularities are mobile for time-dependent systems such as 
occur in the transient response of devices or in short channel devices especially in the presence of fluctuations.

It was shown in [1] that the computed carrier flow in open quantum dots may be described in terms of meandering open orbits which separate regions of trapped flows around vortices. It was conjectured in [1] that the computed carrier flow in open quantum dots may be described in terms of meandering open orbits which separate regions of trapped flows around vortices. It was conjectured in [1] that the topology of the flow may be derived $a b$ initio from a simple geometrical ansatz in which the classical ballistic trajectories are first computed and then all crossings are replaced by hyperbolic flow separation and for which all trapped flows form quantised closed orbits. In the present paper we analyse and extend this picture to a computational methodology for developing quantum hydrodynamic descriptions of flows in the presence of quantised vortices. The outcome is strongly reminiscent of a primitive string theory.

\section{SINGULARITY STRUCTURE}

The steady flow corresponding to (2) and (3) is described by two autonomous coupled equations for the particle position and velocity, which for $2 \mathrm{D}$ correspond to:

$$
\frac{d x}{d t}=v_{x}=m^{-1} \frac{\partial S}{\partial x} ; \quad \frac{d y}{d t}=v_{x}=m^{-1} \frac{\partial S}{\partial y}
$$

The autonomous system (5) describes velocity streamlines or trajectories, which cannot cross because the wave function is single-valued. The topology of the velocity flow may be established by using stability analysis to characterise the flow in the vicinity of the fixed points. There are two classes of singularity in the flow. Linear stability analysis around the velocity nodes, points $\mathbf{x}_{0}$ for which $\mathbf{v}=0$, reveals that for zero magnetic field the local flow pattern is hyperbolic; for non-vanishing magnetic field there occur in addition closed elliptical orbits due to the Lorentz force. The hyperbolic fixed points (saddle points) act to seperate the flow, whereas the closed orbits surrounding fixed points which are centres correspond to Lorentz vortices. A second class of vortex flow occurs even in the absence of a magnetic field when $\nabla \times \mathbf{v}=0$ everywhere except at the nodes in the amplitude of the wave function $[1,5]$. At the amplitude nodes the phase and hence the velocity is not defined. This is a purely quantum effect. The closed orbital motion around the centre of the quantum vortex is due to the quantum potential $V_{Q}$ (see Eq. (3)), which in the vicinity of points where $\sqrt{\rho}=0$, may exhibit a $\left|\mathbf{x}-\mathbf{x}_{0}\right|^{-2}$ dependence leading to closed orbits. In the case of the 2D open quantum dot structures of Figure 1 we therefore expect three classes of flow: (i) open orbits which connect the entry and exit leads of the dot; (ii) hyperbolic orbits which link to the saddle points or terminate at hard wall boundaries; (iii) Lorentz and quantum vortices. In the sequel we shall ignore the Lorentz vortices by setting $B=0$. The wave function may be reconstructed in the vicinity of the singular points from small signal analysis of the flow and the polar form $\Psi(\mathbf{x}, t)=R(\mathbf{x}, t) \exp [i S(\mathbf{x}, t) / \hbar]$.

\subsection{Ab Initio Quantum Hydrodynamics}

Numerical studies of the rectangular open quantum dot [1], essentially a double aperture device, reveal that, standing wave patterns are formed upstream of the first aperture and within the square dot itself. At the first aperture a strong propagating wave is injected which is approximately confined to a ray path of width determined by the aperture and which involves multiple reflections of the boundary walls before reaching the exit aperture. The reflecting ray paths interfere with the standing wave in the dot to form microvortices as with the upstream case in the single aperture. In addition, new and larger vortices form where the ray path loops back on itself due to reflections at the walls. At high wave vectors this is the dominant feature. The origin of the large 

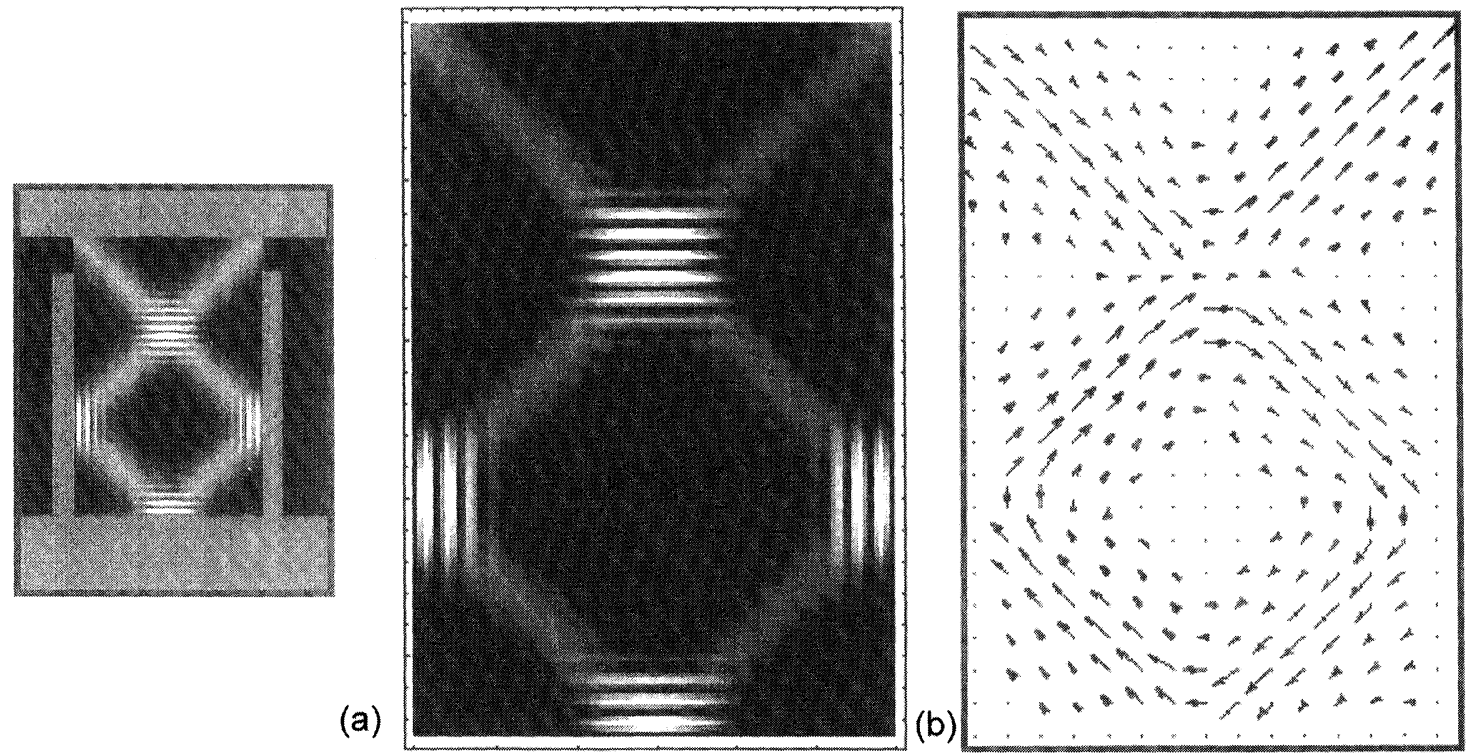

FIGURE 2 The computed flow of a long wave packet in an open rectangular dot. (a) The probability density; (b) the velocity field.

vortices may be seen in Figure 2 where we show a simple reconstruction based on evolving an initial injected long wave packet with a plane wave motion along the direction of the packet and a standing wave in the perpendicular direction. Neglecting dispersion, the evolution of the wave packet involves multiple reflection off the walls and when the phase condition (4) is satisfied a clear vortex develops in the derived velocity flow (Fig. 2).

To construct the vortices we imagine a classical path formed by a free particle bouncing between the walls of the dot where the injection direction is determined from the incident wave-vector components (Fig. 3a). Because quantum trajectories cannot cross, the quantum case is derivable qualitatively from the classical cases by letting the classical streamlines repel each other near crossing points: bifurcation of the flow occurs. The resulting flow topology contains hyperbolic and vortex flows as shown in Figure $3 b$. This result is confirmed by analytical and numerical calculations. The net effect is that the complex classical ray trajectories associated with the quantum ray propagation separates the flow into localised flow
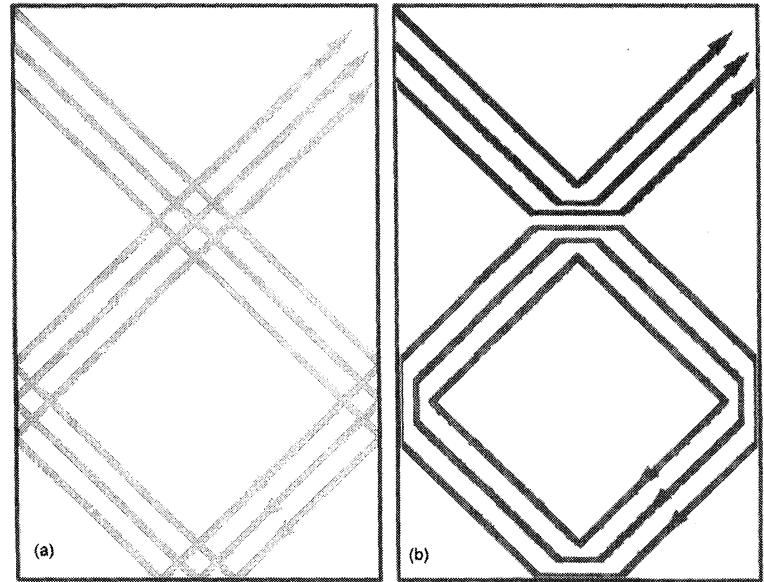

FIGURE 3 Classical ray paths corresponding to Figure 2. (a) Purely classical: path crossing displayed. (b) Quantum case: paths cannot cross: bifurcation occurs.

around vortex centres and meandering open orbits which thread between them. Typically there are several open orbit paths some with minimum traversal times, others with very long paths and hence long traversal times. However, this procedure is qualitative and we turn now to a quantitative algorithm. There are two possible routes: (a) develop a field theory of self-avoiding 
streamlines; (b) develop a particle theory of interacting trajectories. We follow the latter here.

\section{MATERIAL FLUID MODEL}

A classical trajectory is defined as the path followed by a real particle moving through space; the particle is at the head of the trajectory. A simple representation of the quantum flow (quantum trajectories) is obtained by representing each stream line as the path of a stream of noninteracting virtual particles moving classically within the system and distributed according to the probability density $\rho$. If a real particle is located somewhere in space, it is then possible to reconstruct its history by following the streamline back in time. This picture carries precise predictive power but depends upon being able to compute the streamline in the first place from a wave function. To see if this approach will work without recourse to the wave function we might calculate the evolution of a finite bundle of classical particle paths corresponding to an initial set of positions and momenta consistent with the initial wave function (for example for injection into the dot). This is shown in Figure 3 but no interference or vortex motion occurs and indeed the trajectories cross. To extend the model we now let the virtual particles interact through a repulsive hard sphere interaction, which prevents trajectory crossing where two virtual particles collide. If a finite number of particles are used we must maintain commensurability of position to ensure that all
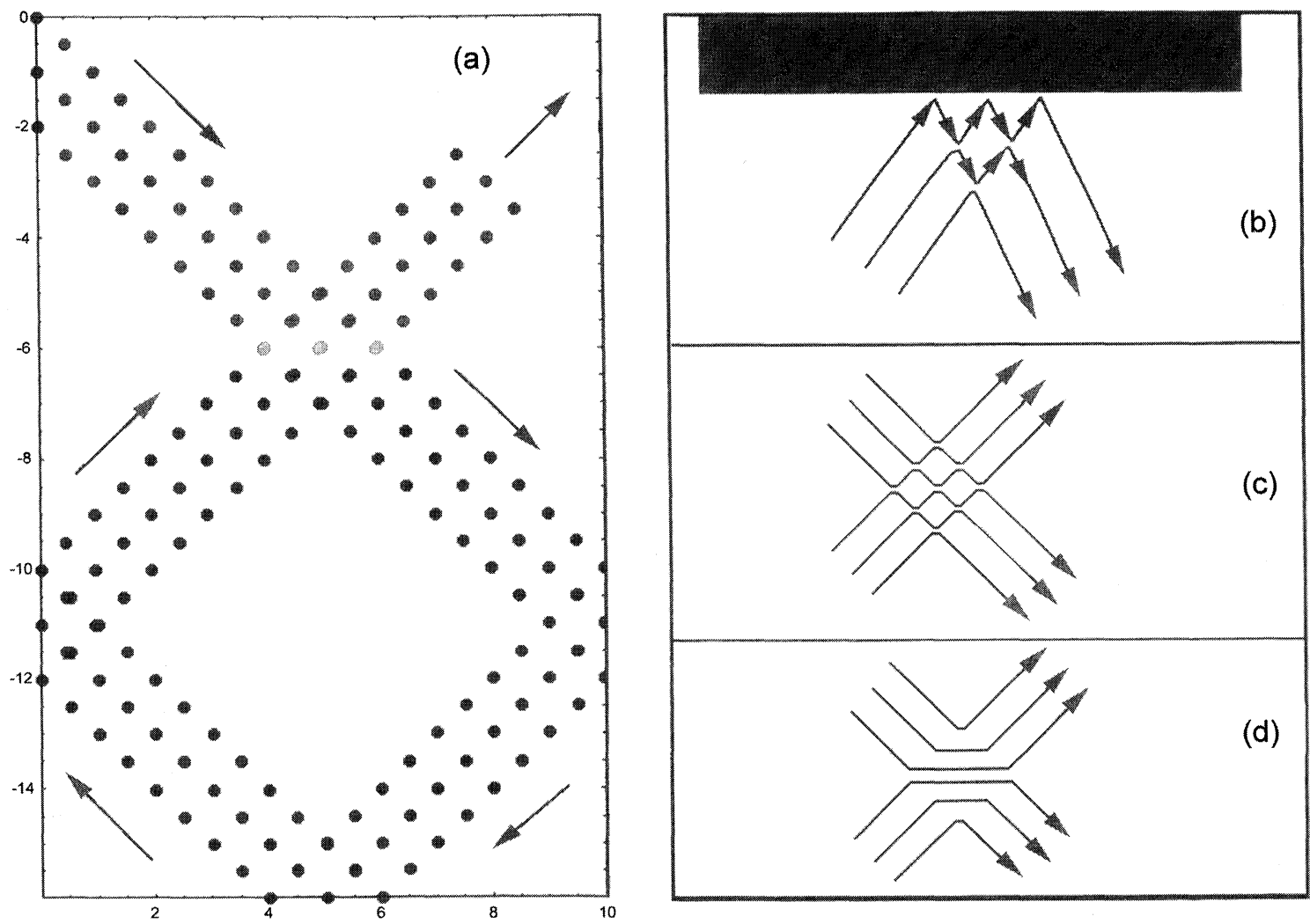

FIGURE 4 Spatially correlated virtual particle model corresponding to Figure 2 under vortex forming conditions (commensurability of virtual particle spacing with closed path length). (a) Positions of virtual particles shown a short time after the flow has developed. (b) Enlargement of interaction of three streams of virtual particles at the wall reflection. (c) Enlargement of interaction of three streams of virtual particles at a cross over region. (d) Flow separation in limit of large number of virtual particle streams. 
crossing trajectories are used we must maintain commensurability of position to ensure that all crossing trajectories are deflected. In the present case the virtual particles are initially out one wavelength $\lambda=\mathrm{h} / \mathrm{mv}$ apart along the path and the streams are separated initially by the corresponding lateral wavelength. Figure 4 illustrates the effect of three such virtual particle streams reflecting off a hard wall (Fig. 4a) calculated by the algorithm. An enlargement (Fig. 4b) shows that the reflection off the wall involves the upper leading particle bouncing off the wall but colliding later with the leading particle in the second stream resulting a return to the wall and a further reflection sequence. As we increase the number of particle streams the net effect is for the lower edge stream of particles to reflect well before the wall, whilst the other trajectories follow a path running parallel to the wall before reflecting. This agrees well with the a posteriori results. Similarly, collisions between particles where trajectories cross show clear flow bifurcation and the appearance of a vortex provided the closed path of each vortex line is commensurable with the particle spacing. This condition is

$$
\begin{gathered}
\oint \frac{d s}{\lambda}=n \Rightarrow \oint \mathbf{k} \cdot d \mathbf{r}=2 \pi n \Rightarrow \oint \hbar \mathbf{k} \cdot d \mathbf{r}=n h \\
\oint m \mathbf{v} \cdot d \mathbf{r}=n h
\end{gathered}
$$

Conditions (6) correspond to the phase condition (4). If (6) is not satisfied, the long-time evolution of the virtual particles leads ultimately to a stationary distribution in the lower part of the dot and an open set of orbits entering and leaving the dot in the upper half.

\section{STRING FLUID MODEL}

The model described in Section 3 is very unstable and of poor practical application. It is also crucial to build in the coherence of the wave function via the spatially correlated initial virtual particle distribution. However, the algorithm may be stabilised by a string model (Fig. 5a) in which each virtual particle is bonded into a string of virtual particles, each of which, is bound by a nearest neighbour attractive potential at large particle separation but has a repulsive core at small separation (Fig. 5d). The model interaction potential for neighbouring virtual particles at $\mathbf{x}_{\mathbf{j}}$ and $\mathbf{x}_{\mathbf{j}+1}$ is chosen as:

$$
V=\sum_{k} \alpha g\left(\mathbf{x}_{k}\right) g\left(\mathbf{x}_{k+1}\right)\left(\frac{1}{g\left(\mathbf{x}_{\mathbf{k}}\right)}-\frac{1}{\left.g(\mathbf{x})_{k+1}\right)}\right)^{2}
$$

where the functions $g\left(x_{j}\right)$ are generalised density functions with a broadened $\delta$-function characteristic describing the smearing out of the virtual particles along the string. String interactions may be modelled by two processes: string repulsion (Fig. 5b) along the bodies of two strings; bond breaking leading to bifurcation of the flow when a string head encounters a string body (Fig. 5c). This model has real computational power and contains several new features. We note first that the virtual particle interactions lead to waves along the string. The parameter $\alpha$ may be chosen to give the familiar quantum relation between wave vector $\mathbf{k}$ for these waves and the particle velocity. The commensurability needed to obtain the quantisation of circulation (a closed string) has its origins here. In the continuum limit the inter-particle potential may be related to the quantum potential. Denoting the average density of virtual particles by $\rho(\mathrm{x})$ in the continuum limit we obtain:

$$
\begin{gathered}
\mathbf{x}_{k} \rightarrow \mathbf{x}, \quad \mathbf{x}_{k+1} \rightarrow \mathbf{x}+\delta \mathbf{x}, \quad \alpha|\delta \mathbf{x}| \rightarrow \beta \\
V \rightarrow \int d^{3} x \rho \beta\left(\frac{\nabla \rho}{\rho}\right)^{2}
\end{gathered}
$$

By choosing the parameter $\beta$ appropriately (7) becomes

$$
\int \frac{\hbar^{2}}{8 m} \rho^{-1}(\nabla \rho)^{2} d^{3} x
$$

The effective Lagrangian for this model may be represented as: $\mathrm{L}=\int d^{3} x L$ where the Lagrangian density is written in terms of the 


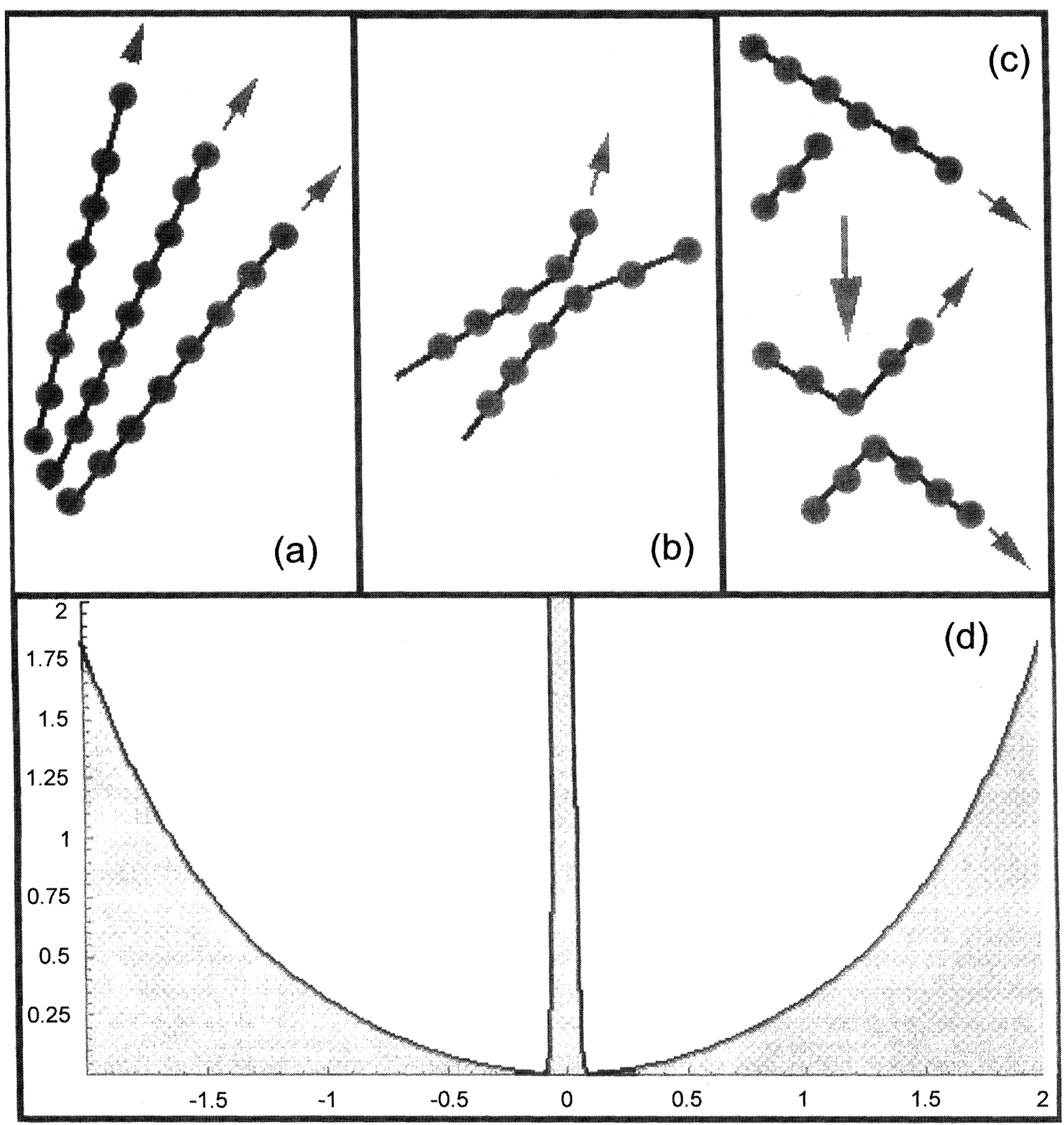

FIGURE 5 The string model. (a) String bundle model. (b) String repulsion. (c) String collision: bond breaking. (d) Effective interparticle interaction potential.

classical action $\mathrm{S}$ as

$$
\begin{gathered}
L=R^{2}(\mathbf{x}, t)\left\{\frac{(\nabla S(\mathbf{x}, t))^{2}}{2 m}+\frac{\hbar^{2}}{2 m}\left(\frac{\nabla R(\mathbf{x}, t)}{R(\mathbf{x}, t)}\right)^{2}\right. \\
\left.+V(\mathbf{x}, t)+\frac{\partial S(\mathbf{x}, t)}{\partial t}\right\}
\end{gathered}
$$

and we have written $\rho=\mathrm{R}^{2}$. The Lagrangian equations of motion are easily obtained for the two fields $\mathrm{R}$ and $\mathrm{S}$ and correspond to Eqs. (1) and (2) consistent with the Schrödinger equation. For a closed string it is essential in this model that Eq. (6) holds. Thus the string model reduces to the conventional Schrödinger picture in the 
continuum limit. It is also relatively easy to calculate the adiabatic evolution of bound states within the string model for example by moving the hard wall boundaries of a stationary confined system.

\section{DISCUSSION AND CONCLUSIONS}

We have outlined a route towards an effective particle representation of simple quantum systems which resemble a self avoiding walk problem or a string theory. The virtue of the model is that for the first time it possible in an $a b$ initio calculation to predict vortex formation and the occurrence of all the topological features of quantum flows. The inter-particle potential plays a similar role to the quantum potential $\mathrm{V}_{\mathrm{Q}}$ in a posteriori quantum hydrodynamics but the incorporation of coherence via the correlated particles in the strings provides the basis for describing interference phenomena and the velocity circulation quantisation. It is clear that practical difficulties remain to be resolved but the full string model in the non-continuum limit holds considerable promise. Furthermore dissipative processes are easily incorporated followin the prescription outlined in [4]. The formalism described here has described coherent quantum transport but provides a basis for the onset of phase breaking processes if a non-Hermitean quantum Hamiltonian is used to derive the string equations of motion.

\section{References}

[1] Barker, J. R., Akis, R. and Ferry, D. K. (2000). Superlattices and Microstructures, 27, 319-325.

[2] Barker, J. R. and Ferry, D. K. (1998). Semiconductor Science and Technology, 13A, 135-139.

[3] Barker, J. R. (1998). Extended Abstracts, 1998 Sixth International Workshop on Computational Electronics (IWCE-6) IEEE Catalog No. 98EX116, pp. 1-4.

[4] Barker, J. R. and Watling, J. (2000). Superlattices and Microstructures, 27, 347-351.

[5] Hua Wu and Sprung, D. W. L. (1994). Phys. Letters A, 196, 229. 

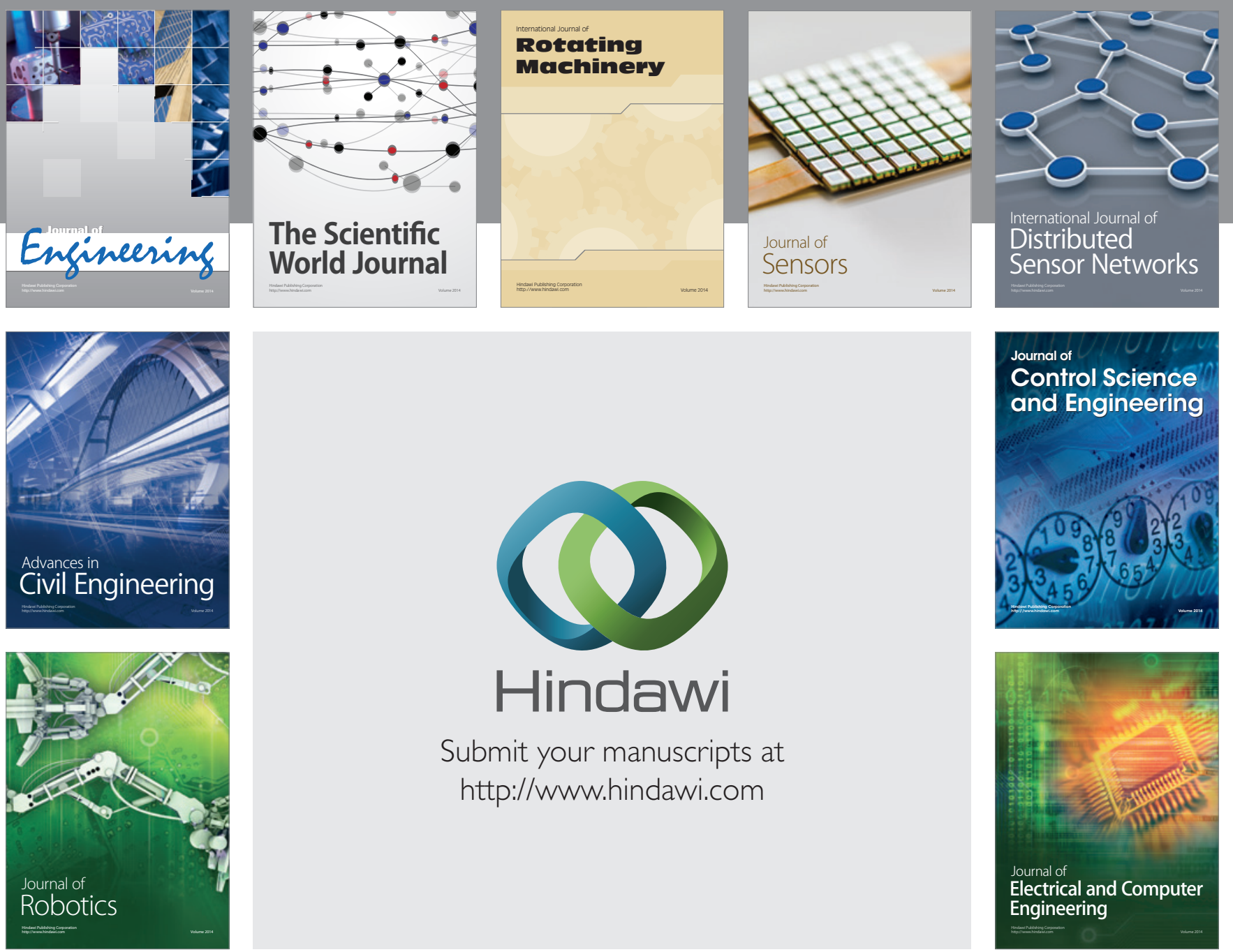

Submit your manuscripts at

http://www.hindawi.com
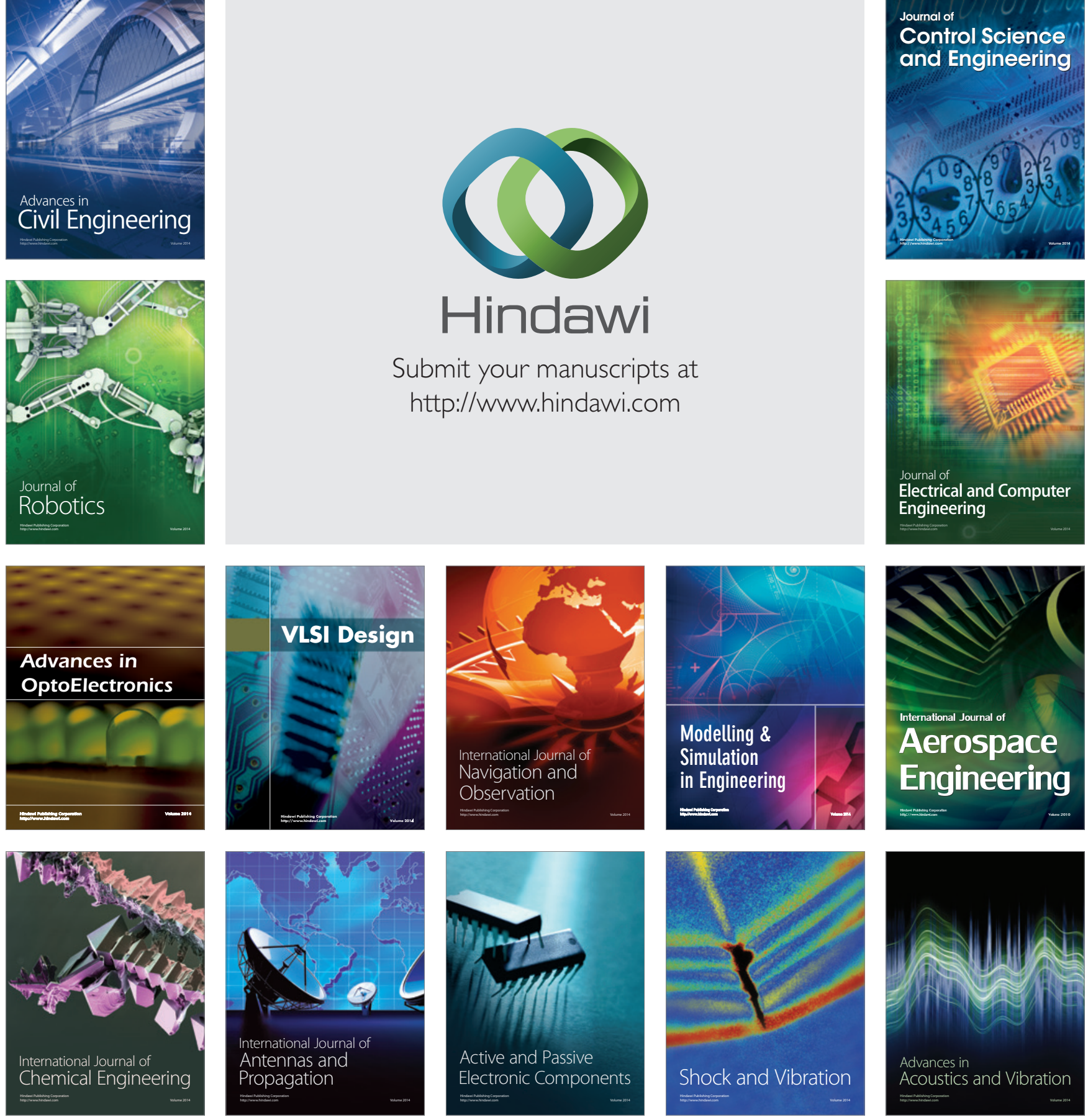